\title{
Texture Deviation Mapping Based on Detail Enhancement
}

\author{
Wen-hua Qian, Dan Xu, Kun Yue, Zheng Guan, Yuan-yuan Pu
}

Department of Computer Science and Engineering, School of Information Science and
Engineering,

Yunnan University, Kunming 650091, China

E-mail: qwhua003@sina.com

Keywords: texture deviation mapping; filter; detail enhancement; artistic appearance

\begin{abstract}
To provide with an effective technique for non-photorealistic rendering for computer generated images with artistic appearances from 2D images motivates our work in this paper. The methods proposed in this paper are inspired by the image deviation mapping constructed from a single texture background image. We establish our method for obtaining artistic appearances taking the deviation mapping as the underlying basis. Based on the simple linear filtering convolution operation, which is well suited of progressive coarsening of images and for detail extraction, and the image's detail, such as edge and tone can be preserved in the final artistic appearance. This method has the exact computational complexity, and this technique is easily to implement and the rendering speed is fast.
\end{abstract}

\section{Introduction}

In the past few years, computer-based artistic style creation has attracted much interest for its wide application in the real world. Non-photorealistic rendering (NPR) is the subject of intense debate for representative methods of artistic style creation. The NPR methods transform an image into specific artistic appearance such as freehand drawing [1], abstraction effects [2] etc., or, more generally in a style other than realism. A new artistic appearance can be synthesized from the existing images, or rendered from a surface model. Because of a pixel in an image contains color information RGB and additional transparency component a, many previous NPR works to paint artistic works use this four components (RGBa). In addition, some researcher rendered an image using the lighting conditions to generate the NPR artistic appearance. These methods use scenes or objects when the geometrical and photometric models are known. However, the models are very difficult to obtain in practice sometimes.

Liu present a technique called "deviation mapping" method to generate images with artistic appearances[3]. In his method, the deviation angle information can be generated from a single background image. Liu's method synthesized images exhibit some artistic appearances. However, during the deviation mapping, because of gamut deviation mapping and the ambient light is constant coefficients, some tone and detail such as edge of source image will be discarded or neglected.

Based on the Liu's method, we introduce a new way to construct edge and tone preserving image decompositions in the final artistic appearance. We advocate the use of an alternative edge preserving smoothing filtering operator, based on the simple linear filtering convolution operation, which is well suited of progressive coarsening of images and for detail extraction. In addition, this technique is easily to implement and the rendering speed is fast. Compared with the other NPR artistic generating methods [4,5], the main advantages of this paper can be summarized as follows: (1)Some details information such as edge and tone can be preserve and appear in the final artistic appearance images.(2)Compared with the deviation mapping generation and bump map, the computation complexity is much cheaper.(3) By the parameters adjusting, our method can obtain some other NPR artistic effects and this will enlarge the fields of NPR artistic appearances in the final works. 


\section{Related Work}

The technology of texture mapping is a famous method for computer generates artistic appearances. Ashikhmin used texture synthesis technology and mapped the natural texture to some other background, and generated some artistic appearances [4]. However, these methods require a high calculate to achieve satisfactory speed. Sometimes the implement and rendering speed is fast, but at the expense of quality degradation. Liu proposed a simple and fast image synthesis methoddeviation mapping [3]. From a background image, this method generated deviation angles of a virtual surface. The virtual surface was then painted with a foreground image and illuminated to generate the final artistic results. It has been applied to generate several artistic effects, including color embossment, cashmere painting, and textile painting. However, this method neglects some detail information such as tone and edge, and sometimes these details information is very important to the final artistic effects.

Fortunately, the technique of image enhancement can be applied to retain edge and other details of foreground. Several edge-preserving smoothing operators have been used in computational photography over the last years. Tomasi applied bilateral filter technique in computer graphics and non photorealistic rendering [6], but this method is limited in its ability to extract detail at arbitrary scales. Yang et al cast the filtering problem as a vector-mapping approximation and solve it using a support vector machine. This method is constructed an edge-preserving filter which has very similar response to the bilateral filter [7]. Hu et al utilized bilateral filter combined with adaptive tonemapping method to improve image quality. Bilateral filter is used to decompose image into different layers, and only large-scale layer image is enhanced by using adaptive tone mapping. Then the details of the original image can be preserved [8].

A general linear filter translation variant filtering process expressed as a weighted average:

$$
\text { Output }_{i}=\sum_{j} W_{i, j} * \text { Input }_{j}
$$

where $\mathrm{i}$ and $\mathrm{j}$ are pixel indexes. $\mathrm{W}_{\mathrm{i}, \mathrm{j}}$ is the filter kernel [6]. Because this filter cost much time to calculate convolution operation, this method requires a high quantization degree to achieve satisfactory speed. To preserve or enhancement the edge detail in the final artistic appearances, based on the method of Liu's deviation mapping, this paper will apply linear image filter to obtain NPR artistic effects.

\section{Edge-preserving of Texture Deviation Mapping}

The bump mapping approach is widely used in the texture synthesis and computer graphics. Different from the bump mapping, the artistic appearance deviation map is derived from a single 2D image and the texture deviation mapping need not any 3D rendering technique but only for image processing[3]. If lighting conditions are given, each pixel in the texture deviation map image can be directly represented as a single deviation angle. Finally, the deviation map can be regarded as a virtual geometry image based image representation or a virtual surface. Compared with the course of bump map generation and mapping, the computation complexity of deviation mapping is much simpler and cheaper.

Phong Illumination Model. If information of surface model is known, the basic technique of Phong shading model can be used to calculate the intensity of each pixel in the images. Let Intensity is value of intensity in the result image, Diffuse and Specular represent the diffuse illuminate and the specular-reflection illuminate [9]. Eq.(2) describe this Phong model:

$$
\text { Intensity }=\operatorname{Diffuse}(L, N)+\operatorname{Specular}(V, R)^{*} n
$$

Based on the Eq. (2), the Eq. (3) describe the more detail in this illumination model [9].

$$
I_{l}=I_{e l} K_{e} V_{d l}+I_{p l}\left[K_{d} V_{d l} \cos \alpha+S \cos ^{m} \beta\right]
$$


$I$ is intensity of the result image, $\alpha$ is the angle between the lighting direction $(L)$ and the normal $(N)$ of the virtual object surface, $\beta$ is the angle between the viewing direction $(V)$ and the reflecting direction $(R), l$ is one of the color channel. Ie is the ambient light, $V d$ is the information of color in the foreground image, which represent the virtual object surface, $I p$ is the lighting color, $m$ is constant coefficients. $S$ can be set to a constant value, which is the material's specular-reflection coefficient. We have to specify all of these parameters in this formula.

Artistic Appearance Deviation Model. The deviation map Pian $(p, q)$ is derived from texture background image, in which each pixel has a deviation angle $\alpha(p, q)$ determined by the intensity of the background image at its corresponding pixel. Let $E(p, v)$ is the result artistic image, $\operatorname{Pian}(p, q)$ is the deviation map image, $F(p, q)$ is the image after foreground's filter, deviation mapping can use Eq.(4) to execute. This formula is a simplified form of Eq. (3). Ia is ambient light, $k a, k s$ and $m$ are constant coefficients:

$$
E(p, q)=I_{a} K_{a}+F(p, q) \cos (\operatorname{Pian}(p, q))+K_{s} \cos ^{m}(\operatorname{Pian}(p, q))
$$

This artistic deviation mapping method is a simplified relighting procedure and the result is good. Suppose the foreground image $F(p, q)$ is painted on a plane that is orthogonal to our viewing direction (V). After the normal at each pixel $(p, q)$ is deviated by the angle $\alpha(p, q)$, and the imaginary virtual surface can be mapped to the background image. Eq.(4) is applied to compute the new color information for each pixel. The foreground image is used as the diffuse color of the material. Because the exact 3D vector expression of the normal is still not known, this can be seen as a virtual surface.

Liu's approach uses the foreground image to map in the background texture image. However, during the deviation mapping process, some detail information such as edge will be neglected, and the edge always is the best important information in the artistic effects. So, this paper improved Liu's method, and we advocate the use of a filter operator to preserve the image's edge.

The technology of image enhancement can be applied to smooth the image, reduce noise and preserve the edge detail. The key edge preserving filter is a local linear model between the foreground image $S(p, g)$ and the filter output $F(p, q)$. Assume the output $F(p, q)$ is a linear transform of foreground $S(p, g)$ in a window $\omega_{k}$ centered at the pixel $k$ :

$$
F(p, q)=a_{k} S(p, g)+b_{k}
$$

where $a_{k}, b_{k}$ are linear coefficients in $\omega_{k}$. We uses a square window of a radius $r$. If foreground image has an edge, because $F^{\prime}(p, q)=a_{k} S^{\prime}(p, q)$, then the output $F(p, q)$ has an edge after filter operation. The important key to the Eq.(5) is ensuring value of the coefficient $a_{k}$ and $b_{k}$. Because this linear model will be used to all local windows in the entire image, after calculating $a_{k}, b_{k}$ for all patches $\omega_{k}$ in the image, the filter results $F(p, q)$ can be computed using[10]:

$$
F(p, q)=\bar{a}_{i} S(p, q)+\bar{b}_{i}
$$

where $\bar{a}_{i}=\frac{1}{|\omega|} \sum a_{k}$ and $\bar{b}_{i}=\frac{1}{|\omega|} \sum b_{k}$. Because the coefficient $\bar{a}_{i}, \bar{b}_{i}$ are the output of an average filter, their gradients should be much smaller than that of input image near strong edges.

For example, Fig.1 shows our linear filter results. If the input image is Fig.1(a), Fig.1(b) is the filter output using the coefficient $a, b$; and the Fig.1(c) is the filter output using the average filter coefficient of $a, b$. Because the filter has the local operation property, the edge in the Fig.1(b) is vague and the edge in the Fig.1(c) can be preserved better than the result of Fig.1(b). 


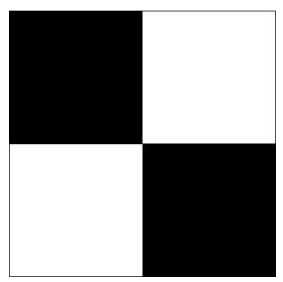

(a)

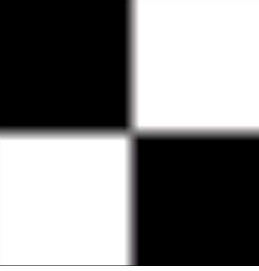

(b)

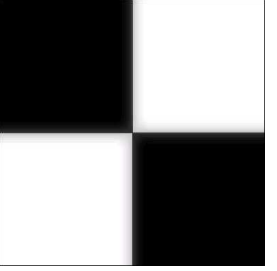

(c)

Fig.1 Filter Results. (a) Input image; (b) Filter results $(a, b)$; (c) Average filter $\operatorname{results}(\bar{a}, \bar{b})$

On the other hand, the parameter Ia of Liu's is only one value which is set up in advance, then this ambient light is no changed during the mapping. However, this light value is very important to the final appearance. So, we adjust this parameter through light supplement of background image. If the initial value of $I a$ is $I_{0}$, we get $I a$ through formula (7) depending on the light of target image. $S(p, q)$ is one light value of pixel in the target image, ceil represents the integral operation, and the parameters $c 1, c 2, c 3$ can be set in advance, we set these parameters $c 1=2, c 2=3, c 3=6$.

$$
I_{a}(p, q)=\left\{\begin{array}{cc}
I_{0}+\operatorname{ceil}\left(\frac{S(p, q)}{c 1}\right) & 0<S(p, q)<80 \\
I_{0}+\operatorname{ceil}\left(\frac{S(p, q)}{c 2}\right) & 80<=S(p, q)<160 \\
I_{0}+\operatorname{ceil}\left(\frac{S\left(\frac{p, q)}{c 3}\right)}{23}\right. & 160<=S(p, q)<255
\end{array}\right.
$$

O(N) Time Computational Complexity. The main computation involved in our method is the Phong illuminate model of deviation map and the filtering of the exponentiation of the original image. This course is very fast because the operations involved are very simple, and this operation has an $\mathrm{O}(\mathrm{N})$ computational complexity. Then, the main time cost is the filter operation. The filtering process in Eq.(6) is a translation variant convolution. When the filtering kernel becomes larger, our method's computational complexity will increase. During the average filter, all the summations in Eq.(6) are box filters. The technology of Integral Image technique [11] gave a method to calculate the output of a box filter, and this filter method has an $\mathrm{O}(\mathrm{N})$ time exact computational complexity. We apply integral image to calculate this box filter. The filter value at any point $(x, y)$ in the summed area table is just the sum of all the pixels above and to the left of $(x, y)[11]$, then:

$$
I(x, y)=\sum_{x^{\prime} \leq x, y^{\prime} \leq y} i\left(x^{\prime}, y^{\prime}\right)
$$

In addition, through a single pass over the image, the summed area table can be calculated efficiently. When the summed area table has been computed, the task of evaluating any rectangle can be accomplished in constant time. Because the pixel only scanned once in the image, the computational complexity of integral image is $O(N)$.

\section{Experiment Results}

To verify the feasibility and effectiveness of our proposed methods in this paper, we implemented the relevant algorithms and carried our corresponding experiments. Our system works on Intel Pentium(R) M processor $2.40 \mathrm{GHz}$ CPU, 1GB PC Memory, Matlab 7.0. Fig.2 shows the experimental results of the gray and colored artistic mapping surface. Fig.2 (b) (d) (f) (h) are artistic deviation results of Liu's [4], and Fig.2 (c) (e) (g) (i) are deviation artistic of detail enhancement results. Different with the artistic of Liu's, the appearance of this paper can maintain more details such as edge and color contrast. The operations are so fast that it takes sever seconds for the method to synthesis final artistic effect images. 


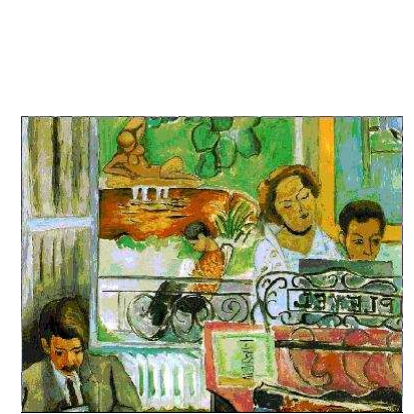

(a)

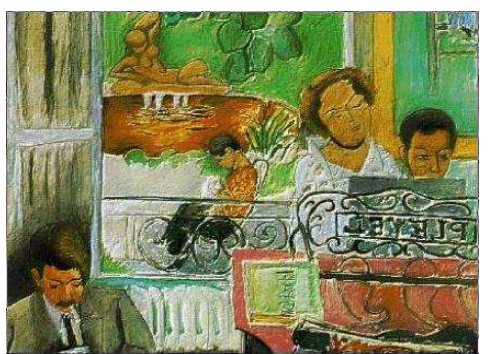

(d)

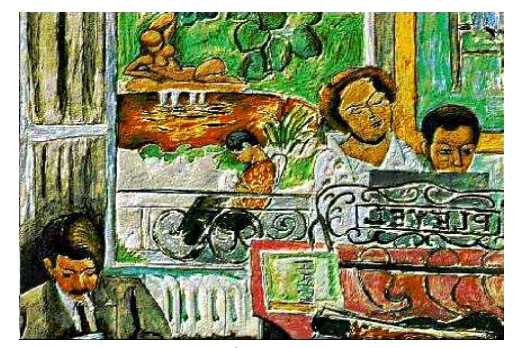

(f)

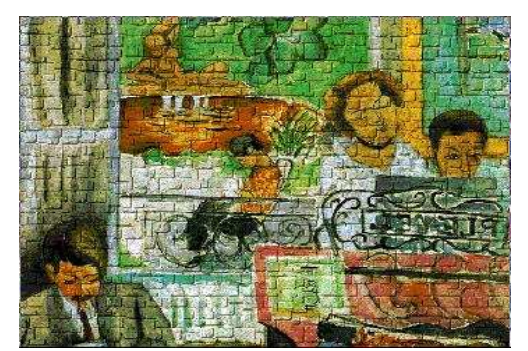

(h)

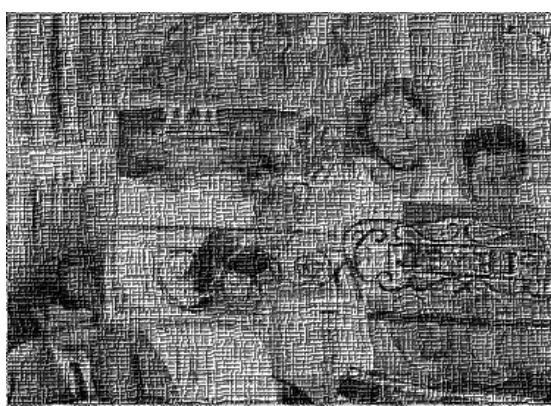

(b)

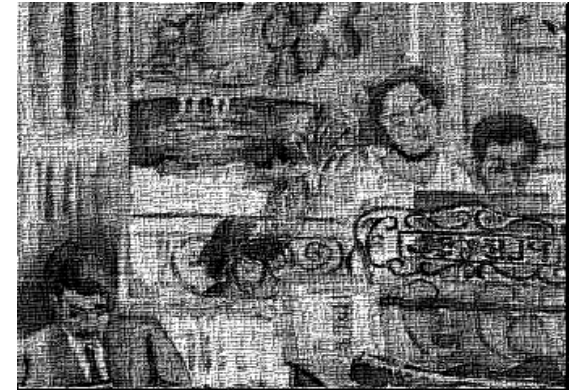

(c)

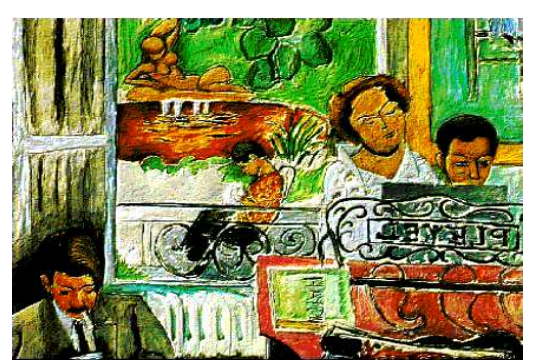

(e)

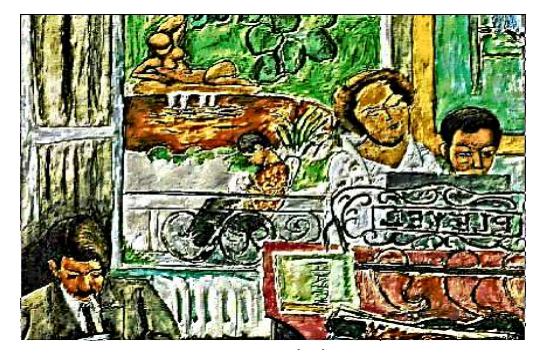

(g)

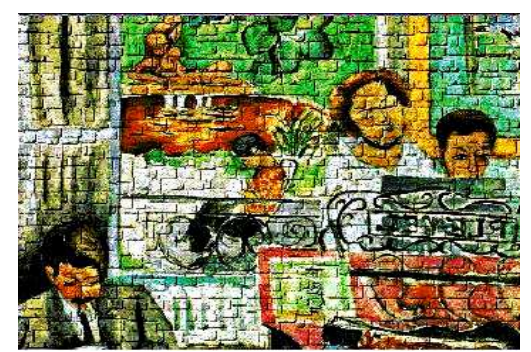

(i)

Fig.2 Deviation gray and colored images by preserving details. (a)Input image; (b) (d) (f) (h) Deviation results of Liu's [4]; (c) (e) (g) (i) Deviation artistic of detail enhancement results.

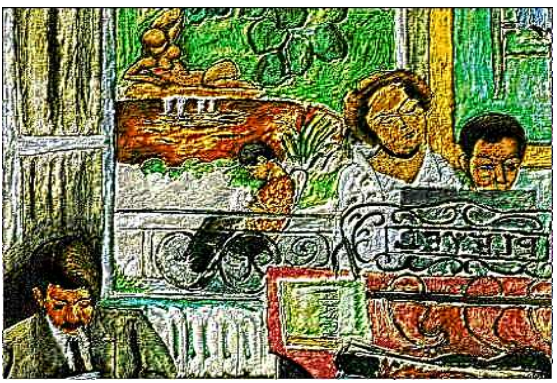

(a)

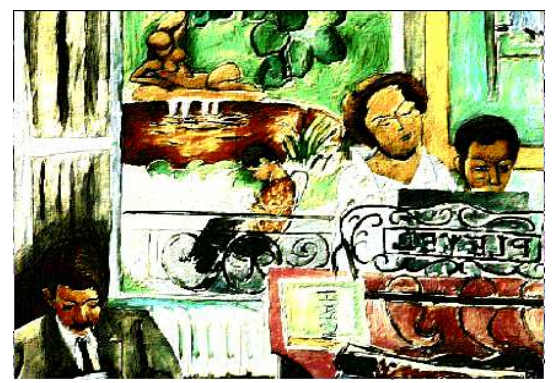

(b)

Fig.3 Other artistic effects by preserving details

It can be seen easily that the experimental results are realistic and they all have some esthetics appearance. During the experiments, we have found that better effects are obtained if the background images are in rough appearances. Therefore, we draw the conclusion that our proposed methods in this paper are reasonable and feasible. And these NPR methods also can generate several other artistic effects, Fig.(3) shows cashmere painting and embossment effects, etc. 


\section{Conclusion and Summary}

In this paper, we proposed a simple and fast $N P R$ technique of artistic deviation mapping appearance. This approach combines two images to generate an NPR artistic effect. From the texture background image's intensity, the deviation map of deviation angles can be generated. Different with the method of Liu [4], this paper advocate the filter operator to preserve the image's detail, such as edge and tone. This method has the exact computational complexity $\mathrm{O}(\mathrm{N})$. Experiments demonstrate that our approach is efficient and effective. Several research issues remain open for future study in computer generated NPR artistic works. Methods for texture representation may be explored to render various classes in 3D models. In addition, conveying a specific mood in the final works is another challenge in the artistic expression.

\section{Acknowledgments}

This material is based upon work supported by the Natural Science Foundation of China Nos. 60663010, the Research Foundation of Yunnan Province No.2010CD024, the Research Foundation of the Educational Department of Yunnan Province.

\section{References}

[1] Y. J. Lee, C.L. Zitnick, M. F. Cohen, ShadowDraw: Real-time user guidance for freehand drawing, ACM Transactions on Graphics (Proceedings of SIGGRAPH 2011), Canada, 2011, pp. $27-34$

[2] J. E. Kypriandis, Image and video abstraction by multi-scale anisotropic kuwahara filtering, Proceedings of the international symposium on Non-photorealistic animation and rendering, NPAR, 2011, pp. 55-64

[3] W. Y. Liu, Z. Hua etc, Artistic Image Generation by Deviation Mapping, International Journal of Image and Graphics, 2001, Vol. 1(4), pp. 565-574

[4] M. Ashikhmin. Synthesizing natural textures, ACM Symposium on Interactive 3D Graphics, March 2001, ISBN 1-58113-292-1, 2001, pp. 217-226

[5] A. Hertzmann, Charles E. Jacobs, Nuria Oliver, et al, Image analogies, Proceedings of the 28th annual conference on Computer graphics and interactive techniques, SIGGRAPH, 2001, pp. $327-340$

[6] C. Tomasi, R. Manduchi, Bilateral Filterng for Gray and Color Images, Proceedings of the 1998 IEEE International Conference on Computer Vision, 1998, pp. 839-846

[7] Q. X. Yang, S. N. Wang, N. Ahuja, SVM for Edge-Preserving Filtering, IEEE Conference on Computer Vision and Pattern Recognition, 2010, pp. 775-782

[8] K. J. Hu, M. Y. Lu, J. C. Wang, T. Hsu, and T.T. Chang, Using Adaptive Tone Mapping to Enhance Edge-Preserving Color Image Automatically, Image and Video Processing, 2010(3), pp. 1-11

[9] B.T., Phong, Illumination for Computer Generated Pictures, CACM,1975,18(6) , pp. 311-317

[10]K. M. He, J. Sun, X. O. Tang, Guided Filter, Computer Vision, ECCV, 2010, (6311) , pp. 1-14

[11]B. Derek, R. Gerhard, Adaptive thresholding using the integral image, Journal of graphics tools, 2007, 12(2), pp. 13-21 